Rev. Biol. Trop., 47(3): 287-308, 1999

www.ucr.ac.cr. www.ots.ac.cr www.ots.duke.edu

\title{
Líquenes folícolas de la Estación Biológica La Selva, Costa Rica: Inventario, comunidades y comparación florística de tipos de vegetación
}

\author{
Robert Lücking ${ }^{1}$ \\ 1 Institut für Pflanzensystematik, Universität Bayreuth, D-95447 Bayreuth, Alemania. Fax: 0049-921-552786. Correo \\ electrónico: rlucking@ hotmail.com
}

Recibido 29-VI-1998. Corregido 11-I-1999. Aceptado 15-I-1999.

\begin{abstract}
A total of 280 foliicolous lichens and 12 lichenicolous fungi was found in an inventory made in the lowland rain forest at La Selva Biological Station, Costa Rica. The species composition reflects $50 \%$ of the world's diversity of foliicolous lichens and is representative for neotropical lowland rain forests. The most common species are Gyalectidium filicinum, Sporopodium leprieurii, Tricharia vainioi, Porina epiphylla, Phyllophiale alba, Trichothelium epiphyllum, Mazosia phyllosema, Tricharia urceolata, and Arthonia leptosperma. Many species show distinct microsite preferences: (1) Characteristic of the shady understory, including Arthoniaceae, Opegraphaceae, Pilocarpaceae, and Trichotheliaceae; (2) characteristic of light gaps, including Gomphillaceae and Ectolechiaceae; (3) characteristic of the canopy, including Asterothyriaceae and Gomphillaceae. Only few species exhibit preferences towards certain leaf types, either dicotyledoneous or palm leaves. The microsite preferences agree with earlier established indices, except for a few species for which modified indices are proposed. Communities resulting from these preferences are illustrated by clustering of phorophytes and associations of foliicolous lichens. The three principal associations correspond to the shady understory, light gaps and the canopy, whereas two subassociations reflect subtle phorophyte preferences. Species diversity and composition are strongly affected by the degree of disturbance of vegetation types at La Selva. Diversity is highest in the primary forest and lowest in young successional stages. The foliicolous lichen flora of open, anthropogenic vegetation resembles that of light gaps or the canopy in the primary forest, while species characteristic of the forest understory disappear.
\end{abstract}

Key words: Foliicolous lichens, Costa Rica, La Selva, inventory, diversity. associations, anthropogenic influence.

La exuberante colonización de la superficie de hojas de plantas vasculares por líquenes y briófitas folícolas, los llamados "epífilos", es un fenómeno particular de bosques tropicales húmedos (Santesson 1952, Richards 1984, Gradstein y Pócs 1989, Sérusiaux 1989, Sipman y Harris 1989, Lücking 1995a). Con respecto a los líquenes, la mayor diversidad en el Neotrópico se encuentra en bosques húmedos de bajura, como en el Norte de Suramérica (Amazonas) o en Costa Rica en los bosques de la vertiente caribeña (Caño Negro, Tortuguero, Hitoy Cerere) y Pacífica Central y Sur (Carara, Corcovado; Liicking 1992a, 1992b, 1995a, 1997a). En un sitio pueden coexistir más de 200 especies, y en una hoja se han encontrado 48-82 especies (Lücking 1995a, 1999a, Lücking y Matzer, in prep.).

Costa Rica es el país con el mayor número de especies de líquenes folícolas reportadas hasta la fecha, con alrededor de 350 de las 550 especies que se conocen nuudialmente (Lücking 
1995a, d). La alta diversidad de zonas de vida que se encuentra en el país ha permitido hacer estudios detallados sobre la distribución de especies con respecto a condiciones ambientales y tipos de vegetación (Lücking 1992a, 1992b, 1995a, 1997b). También ha sido investigada la distribución de especies dentro de un sitio, para establecer preferencias por determinados micrositios que se deben al microclima y a la composición de forófitos. Estos estudios, que se llevaron a cabo en el sendero Botarrama del Parque Nacional Braulio Carrillo, un bosque pluvial premontano, revelaron tres comunidades distintas de líquenes folícolas distribuidas en el sotobosque, en los claros y en el dosel (Lücking 1995b, 1998b, 1998d).

Con base en estos resultados e incluyendo datos de estudios semejantes realizados en otras regiones (Busse 1905, Fitting 1910, Allan 1928, Santesson 1952, Nowak y Winkler 1970, Pócs 1978, Sérusiaux y de Sloover 1986, Sérusiaux 1989, Barillas et al. 1993), se estableció una lista de índices para 270 especies de líquenes folícolas, que permite la bioindicación de zonas altitudinales, estacionalidad, perturbaciones antropogénicas y micrositios (Lücking 1997b).

En vista de lo hecho anteriormente, los objectivos del presente trabajo, realizado en la Estación Biológica La Selva, fueron los siguientes: (1) Hacer por primera vez un inventario completo, incluyendo el dosel, de líquenes folícolas en un bosque tropical muy húmedo de bajura. (2) Aumentar el conocimiento de la biodiversidad que existe en La Selva y establecer una base para un inventario más amplio de líquenes y briófitas. (3) Estudiar la distribución de líquenes folícolas en diferentes micrositios dentro del bosque primario, así como en tipos de vegetación con diferentes grados de perturbación. (4) Comparar los resultados actuales con los obtenidos anteriormente en el sendero Botarrama, para establecer similitudes y diferencias en la composición de especies y la formación de comunidades entre un bosque muy húmedo de bajura y un bosque pluvial premontano pertenecientes al mismo ecosistema, el Parque Nacional Braulio Carrillo.

\section{MATERIALES Y MÉTODOS}

Área de estudio: La Estación Biológica La Selva está localizada en la vertiente Atlántica, al Norte de la Cordillera Volcánica Central en la Provincia de Heredia, Sarapiquí (Fig. 1A). Está conectada con el Parque Nacional Braulio Carrillo por un corredor que incluye un transecto sin interrupción desde La Selva a la cima del volcán Barva. La precipitación excede $4.000 \mathrm{~mm}$ anuales, y la vegetación principal consiste en bosque tropical muy húmedo de bajura, con parches de bosque pantanoso. También incluye vegetación secundaria de diferentes edades y grados de perturbación, además de parcelas de sucesión que permiten estudios comparativos de la dinámica de un bosque tropical (McDade et al. 1994).

El árbol característico de La Selva es Pentaclethra macroloba (Willd.) Ktze (Mimosaceae). En el subdosel se destacan las palmas Euterpe macrospadix Oersted, Iriartea deltoidea Ruíz \& Pavón, Socratea exorrhiza (C. Martius) Wendland yWelfia georgii Wendland ex Burret. Parches de bosque secundario son dominados por Cecropia insignis Liebm., C. obtusifolia Bertol., Pourouma bicolor Martuis (Cecropiaceae), y Rollinia microsepala Standley (Annonaceae). Abundantes en el sotobosque son las palmas Asterogyne martiana (H. Wendl.) H. Wendl., Chamaedorea tepejilote Liebm., Geonoma congesta Wendland ex Spruce y G. cuneata Wendl. ex Spruce. Entre las dicotiledoneas hay que mencionar Ocotea atirrensis Mez \& J. D. Smith ex $\mathrm{Mez}$ (Lauraceae) y Mabea occidentalis Benth. (Euphorbiaceae). Araceas como Anthurium bakeri Hook. f., Dieffenbachia longispatha Engler \& K. Krause, Monstera tenuis K. Koch y Rhodospatha wendlandii Schott ex Engler crecen abundantemente en el suelo y como (hemi)epífitos (Hartshorn y Hammel 1994), todos como forófitos para los líquenes folícolas.

Trabajo de campo: El trabajo de campo se realizó de septiembre 1991 a enero 1992 y de enero a agosto de 1997. Fueron establecidos tres métodos de recolección: (1) Recolección 


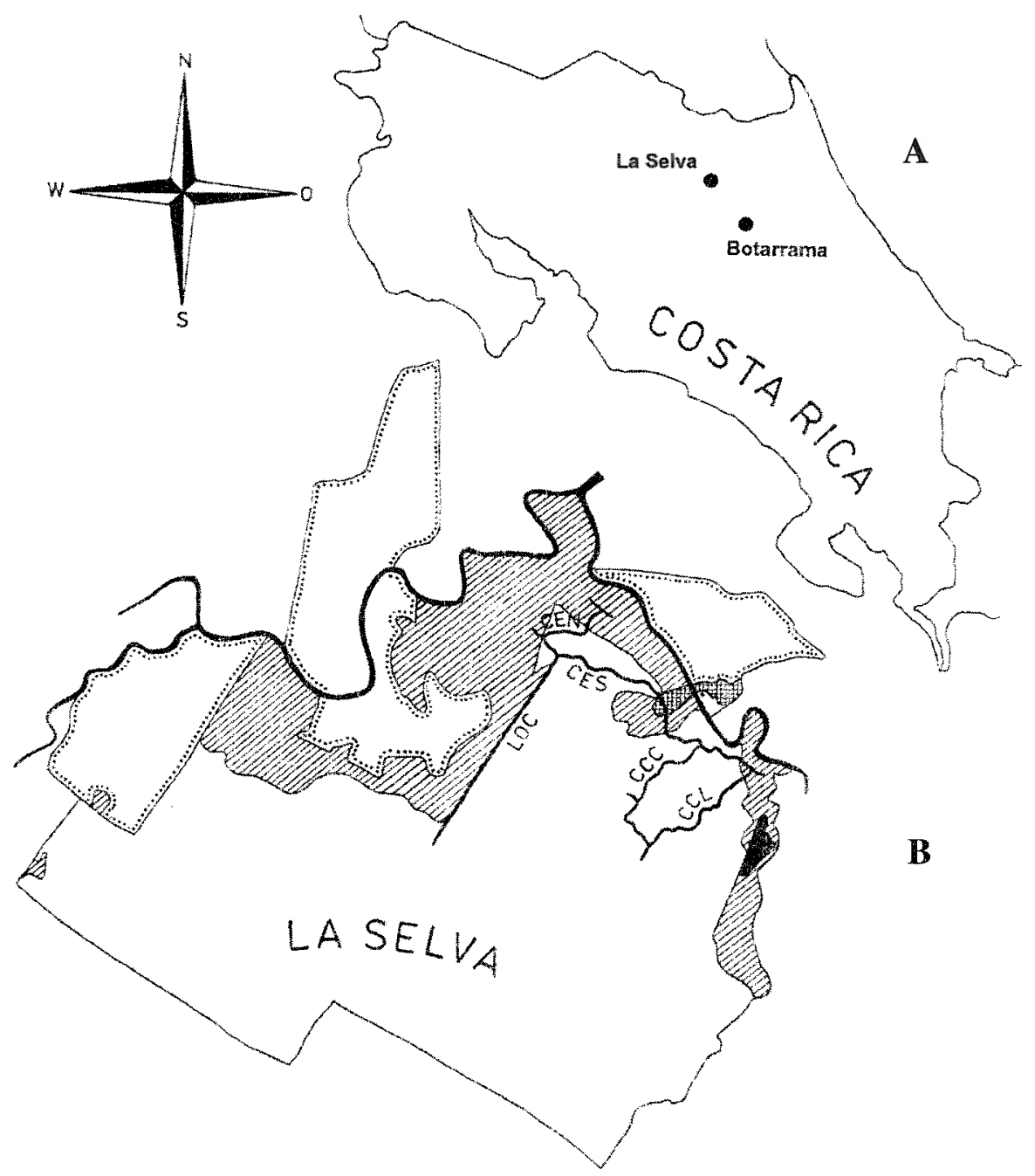

Fig. 1. Situación geográfica del área de estudio, con los tipos de vegetación recolectados y los transectos de los senderos CES, CEN, LOC, CCL y ShO en el bosque primario. Segmentos blancos: bosque primario; segmentos rayados: bosque secundario; segmentos cruzados: vegetación antropogénica; segmentos negros: parcelas de sucesión; segmentos marginalmente puntados: pastos.

general en todo el área de La Selva, con la finalidad de obtenerse el mayor número posible de especies, siguiendo las instrucciónes sugeridas por Lücking y Lücking (1996). (2) Recolección en cinco tipos de vegetacíon, para establecer probables diferencias en la diversidad y composición de especies: bosque primario, bosque secundario, parcelas de sucesión, margen de bosque, $\mathrm{y}$ vegetación antropogénica (Fig. 1B). (3) Recœlección sistemática dentro del bosque primario, para establecer diferencias en la diversidad y composición de especies entre sotobosque, claros y el dosel, y sobre diversos tipos de forófitos. Para este objectivo, fueron seleccionados 100 forófitos, de los cuales 40 pertenecientes al sotobosque, 40 a claros, y 20 al dosel. Dentro del soiobosque y de los claros, cada 20 de los 40 forófitos representaron el tipo de hojas dicotiledoneas de tamaño mesofilo (hoja "normal" según Vareschi 
1980), como se encuentra frecuentemente en la familias Annonaceae (Guatteria), Lauraceae (Ocotea), Meliaceae (Guarea), o Moraceae (Naucleopsis), entre otros, mientras que los otros 20 forófitos representaron el tipo de hoja palmera, típico para las familias Arecaceae y Cyclanthaceae. En el dosel, solamente se tomaron en cuenta hojas dicotiledoneas (Cecropia, Pourouma, Brosimum, Rollinia), ya que estas son mucho más frecuentes que palmas en este micrositio.

La selección de los 100 forófitos fue realizada a lo largo de los senderos CES, CEN, LOC, CCL y SHO, con cuatro transectos puestos en parches de bosque primario (Fig. 1B). Para diferenciar entre sotobosque y claros con respecto a la intensidad de luz relativa ("diffuse site factor"; Anderson 1964), fue utilizada una cámara fotográfica con lente "ojo de pez" y fue medido el tiempo de exposición en comparación con un punto fijado fuera del bosque. Usando una apertura que resulta en un tiempo de exposición de 1/1000 segundos fuera del bosque, del sotobosque fueron considerados forófitos con un tiempo de exposición de 1/8 a 1/15 segundos, lo que corresponde a una intensidad relativa de luz de 1-2\%. En los claros se tomaron en cuenta forófitos con un tiempo de exposición de 1/60 a $1 / 125$ segundos $(=5-10 \%$ de intensidad relativa de luz). En el dosel, fueron seleccionados forófitos con hojas completamente expuestas, lo que corresponde a una intensidad relativa de luz de 70-100 \%. La recoleccion de las hojas del dosel fue realizada escalándose los árboles con la técnica establecida por Perry y Williams (1981) y también mediante cuerdas puestas con una ballesta encima de los árboles. En cada forófito fueron seleccionadas tres hojas de diferentes ramas, y se tomaron en cuenta solamente hojas con una cobertura relativa de líquenes folícolas estimada a por lo menos $20 \%$.

Los 100 forófitos seleccionados de esta manera no representan la abundancia exacta de micrositios y tipos de forófitos en el área de estudio. Por eso, a lo largo de los transectos se estimó también la abundancia actual de micro- sitios y tipos de forófitos: sotobosque vs. claros, forófitos con hojas de tipo dicotiledoneas vs. palmas vs. otros, y el porcentaje de forófitos con cobertura relativa de líquenes folícolas de al menos $20 \%$ en hojas particulares. También se estimó la relación de área entre sotobosque, subdosel y dosel, tomando en cuenta la estratificación del bosque en tres estratos idealizados. Estas estimaciones se tomó como base para proyectar la abundancia actual de líquenes folícolas.

Determinación de líquenes folícolas: $\mathrm{Pa}$ ra la determinación de líquenes folícolas, fueron utilizadas las claves de Santesson (1952), Kalb \& Vězda (1988) y Lücking (1992c, 1997c), además de otras referencias recientes (Farkas \& Sipman 1997). Colecciones representativas de especímenes fueron depositadas en el Herbario Nacional de Costa Rica (CR), en el Instituto de Biodiversidad (INBio), en el herbario de la Escuela de Biología de la Universidad de Costa Rica (USJ), en el herbario de la Estación Biológica La Selva (hb. La Selva), en el herbario del autor (hb. Lücking) y en la exsicata "Lichenes Foliicoli Exsiccati" (Lücking 1997d).

Evaluación de datos: La composición de especies de líquenes folícolas encontradas en cada uno de los 100 forófitos recolectados fue determinada. Para obtenerse la frecuencia de cada especie, se tomó en cuenta el número de forófitos en los cuales esta especie fue encontrada, también considerando la abundancia actual de diferentes micrositios y tipos de forófitos.

Para establecer similitudes en la composición de especies entre forófitos, fue realizado un análisis cluster, usando el método de agrupación de Ward y el índice de similaridad de Sørensen (1948): $\mathrm{S}=2 \mathrm{n}_{\mathrm{a}+\mathrm{b}} /\left(\mathrm{n}_{\mathrm{a}}+\right.$ $\mathrm{n}_{\mathrm{b}}$ ), donde $\mathrm{S}=$ índice de similaridad (con valores entre 0 y 1$), \mathrm{n}_{\mathrm{a}+\mathrm{b}}=$ número de especies de líquenes folícolas en común entre dos forófitos a y $\mathrm{b}, \mathrm{y} \mathrm{n}_{\mathrm{a}} / \mathrm{n}_{\mathrm{b}}=$ número de especies de líquenes folícolas presentes en los forófitos a y b, respectivamente. Para verificarse la existencia de patrones repetitivos, los 100 forófitos fueron separados en dos grupos de 50 individuos cada uno. 
Diferencias significativas en la frecuencia de líquenes folícolas de diferentes micrositios o forófitos fueron comprobadas mediante una prueba de Chi-Cuadrado. Para ilustrar comunidades que resultan de estas diferencias, fue utilizado un análisis de asociaciones (Goodall 1978, Greig-Smith 1983), usando un valor de Chi-Cuadrado estándarizado según Lücking (1999d) para cada pareja de especies posible: $\chi_{\text {stand. }}=\left(\chi_{2} / 100\right)^{1 / 2}$. Este valor tiene variación entre los valores de $-1 \mathrm{y}+1 \mathrm{y}$ indica la co-occurrencia de especies que están significativamente arriba o abajo de lo esperado por su frecuencia. Los datos relacionados con las asociaciones múltiples fueron procesadas en un programa de escala multidimensional para crear un diagrama bidimensional de estas especies.

Utilizando la frecuencia de especies en diferentes micrositios, se establecio un código de preferencia para cada especie, con base en el promedio relativo ("weighted average"; Gauch 1982, Jongman et al. 1995), dando a los micrositios los codigos 1 (sotobosque), 3 (claros), y 5 (dosel; vease Lücking 1997b): CP $=\left(h_{\text {sotobosque }} 1+h_{\text {claros }} 3+h_{\text {dosel }} 5\right) /\left(h_{\text {sotobos- }}\right.$ que $+h_{\text {claros }}+h_{\text {dosel }}$ ), donde $C P=$ código de preferencia y $\mathrm{h}=$ frecuencia absoluta de la espe- cie en un micrositio dado. Estos códigos también fueron utilizados para la comparación con los índices de micrositio propuestos por Lücking (1997b).

Mediante un análisis de componentes principales ("principal component analysis"; Gauch 1982, Jongman et al. 1995), basado en la composición y frecuencia de líquenes folícolas, se hizo una ordinación de los cinco diversos tipos de vegetación, adicionalmente separando el bosque prim rio en sotobosque, claros y dosel. Todos los cálculos estadísticos fueron hechos con el programa STATISTICA 5.0, con excepción de los índices de Sørensen y el análisis de asociaciones que fueron calculados usando programas en Q-BASIC escritos por el autor.

\section{RESULTADOS}

Diversidad y composición de especies: El inventario reveló 280 especies de líquenes folícolas en 54 géneros, 19 familias y 10 órdenes, además de 12 especies de hongos liquenícolas en 7 géneros y 7 familias (Cuadro 1). Sobre los 100 forófitos recolectados en el bosque

\section{CUADRO 1}

Lista de especies de líquenes folícolas y hongos liquenícolas encontrados en la Estación Biológica La Selva, arreglado en orden alfabético por órdenes, familias, géneros y especies. La nomenclatura sigue la monografías de Santesson (1952) y Matzer (1996) y la lista de Farkas \& Sipman (1997), complementado por los trabajos de Lücking y Matzer (1996), Lücking y Sérusiaux (1997), Lücking (1995c, 1997c, e, 1998c), Lücking y Vězda (1998), y Lücking et al. (1994, 1997, 1998a-b). Especies en proceso de publicación: "ined"

List of species of foliicolous lichens and lichenicolous fungi found at La Selva Biological Station, arranged alphabetically with orders, families, genera, and species. Nomenclature follows the monographs of Santesson (1952) and Matzer (1996) and the checklist provided by Farkas \& Sipman (1997), complemented by the papers of Lücking and Matzer (1996), Lücking and Sérusiaux (1996), Lücking (1995c,1997c, e, 1998c), Lücking and Vězda (1998), and Lücking et al. (1994, 1997, 1998a-b). Species in the process of publication are marked ined

Líquenes folícolas autónomos:

\section{ARTHONIALES: ARTHONIACEAE}

Amazononyces farkasiae (R. Lücking) R. Lücking \& Sérus. Arthonia accolens Stirt.

Arthonia aciniformis Stirt.

Arthonia cyanea Müll. Arg.

Arthonia leptosperma (Müll. Arg.) R. Sant.

Arthonia mira R. Sant.

Arthonia orbygniae (H. B. P. Upadhyay) Matzer

Arthonia palmulacea (Müll. Arg.) R. Sant.

\author{
Arthonia trilocularis Müll. Arg. \\ Cryptothecia candida (Kremp.) R. Sant. \\ Cryptothecia sp. A \\ Cryptothecia sp. B \\ Cryptothecia sp. C \\ Cryptothecia sp. D \\ Eremothecella calamicola Syd.
}

\section{ARTHONIALES: OPEGRAPHACEAE}

Mazosia bambusae (Vain). R. Sant. Mazosia dispersa (Hedr.) R. Sant. 
Mazosia longispora $\mathrm{R}$. Lücking \& Matzer

Mazosia melanophthalma (Müll. Arg.) R. Sant.

Mazosia paupercula (Müll. Arg.) R. Sant.

Mazosia phyllosema (Nyl.) Zahlbr.

Mazosia pilosa Kalb \& Vězda

Mazosia praemorsa (Stirt.) R. Sant.

Mazosia pseudobambusae Kalb \& Vězda

Mazosia rotula (Mont.) A. Massal.

Mazosia rubropunctata $\mathrm{R}$. Sant.

Mazosia tenuissima R. Lücking \& Matzer

Opegrapha lambinonii Sérus.

\section{DOTHIDEALES: MICROTHELIOPSIDȦCEAE}

Microtheliopsis uleana Müll. Arg.

DOTHIDEALES: Genera incertae sedis

Caprettia nyssaegenoides Sérus. ined.

Lyromma nectandrae Bat. \&. H. Maia

Lyromma palmae (Cavalc. \& Silva) R. Lücking \& Serus.

\section{GRAPHIDALES: THELOTREMATACEAE}

Chroodiscus australiensis Lumbsch \& Vězda Chroodiscus coccineus (Leight.) Müll. Arg. Chroodiscus neotropicus Kalb \& Vězda

\section{GYALECTALES: GYALECTACEAE}

Coenogonium interplexum Nyl.

Coenogonium interpositum $\mathrm{Nyl}$

Coenogonium linkii Ehrenb.

Coenogonium ciliatum Kalb \& R. Lücking

Dimerella dilucida (Kremp.) R. Sant.

Dimerella epiphylla (Müll. Arg.) Malme

Dimerella fallaciosa (Müll. Arg.) Vězda

Dimerella flavicans Vězda \& Farkas

Dimerella hypophylla Vězda

Dimerella isidiifera R. Lücking

Dimerella lisowskii Vězda

Dimerella cf. lutea (Dicks.) Trevis.

Dimerella minima (Müll. Arg.) R. Sant.

Dimerella siquirrensis R. Lücking

Dimerella vezdana $\mathrm{R}$. Lücking

\section{LECANORALES: BACIDIACEAE}

Bacidia corallifera R. Lücking

Bacidia nigrescens (Müll. Arg.) Vain.

Bacidia psychotriae (Müll. Arg.) Zahlbr.

Bacidina apiahica (Müll. Arg.) Vězda

Bacidina defecta Vězda

Bacidina hypophylla R. Lücking \& Kalb

Bacidina mirabilis (Vězda) Vězda

Bacidina pallidocarnea (Müll. Arg.) Vězda

Bacidina scutellifera (Vězda) Vězda

Bapalmuia lineata R. Lücking ined.

Bapalmuia palmularis (Müll. Arg.) Sérus.

Bapalmuia aff. palmularis (Müll. Arg.) Sérus.

Woessia pseudohyphophorifera R. Lücking \& Sérus.

\section{LECANORALES: COCCOCARPIACEAE8}

Coccocarpia domingensis Vain.

Coccocarpia palmicola (Spreng.) L. Arvidss. \& D. Gall. Coccocarpia pellita (Ach.) Müll. Arg.

Coccocarpia stellata Tuck.

\section{LECANORALES: COLLEMATACEAE}

Leptogium sp.

\section{LECANORALES: ECTOLECHIACEAE}

Calopadia foliicola (Fée) Vězda

Calopadia fusca (Müll. Arg.) Vězda

Calopadia phyllogena (Müll. Arg.) Vězda

Calopadia puiggarii (Müll. Arg.) Vězda

Lasioloma arachnoideum (Kremp.) R. Sant.

Loflammia flammea (Müll. Arg.) Vězda

Loflammia gabrielis (Müll. Arg.) Vězda

Pseudocalopadia mira R. Lücking ined.

Sporopodium antonianum Elix, Lumbsch \& R. Lücking. Sporopodium citrinum (Zahlbr.) Elix, Lumbsch \& Lücking Sporopodium leprieurii Mont.

Sporopodium phyllocharis (Mont.) Massal.

Tapellaria epiphylla (Müll. Arg.) R. Sant.

Tapellaria phyllophila (Stirt.) R. Sant.

Tapellariopsis octomera R. Lücking

\section{LECANORALES: PANNARIACEAE}

Pannaria sp.

\section{LECANORALES: PILOCARPACEAE}

Badimia dimidiata (Babingt. ex Leight.) Vězda

Badimia cf. galbinea (Krempelh.) Vězda

Byssolecania deplanata (Müll. Arg.) R. Sant.

Byssolecania fumosonigricans (Müll. Arg.) R. Sant.

Byssolecania imenocarpa (Vain) Kalb, Vězda \& R. Lücking

Byssolecania variabilis Vězda, Kalb \& R. Lücking

Byssoloma absconditum Farkas \& Vězda

Byssoloma aurantiacum Kalb \& Vězda

Byssoloma chlorinum (Vain.) Zahlbr.

Byssoloma fadenii Vězda

Byssoloma leucoblepharum (Nyl.) Vain.

Byssoloma minutissimum Kalb \& Vězda

Byssoloma subdiscordans (Nyl.) P. James

Byssoloma tricholomum (Mont.) Zahlbr.

Byssoloma vanderystii Sérus.

Byssoloma wettsteinii (Zahlbr.) Zahlbr.

Fellhanera angustispora R. Lücking

Fellhanera aff. angustispora R. Lücking

Fellhanera badimioides R. Lücking, Lumbsch \& Elix

Fellhanera bouteillei (Desm.) Vězda

Fellhanera dictyospora $\mathrm{R}$. Lücking

Fellhanera fuscatula (Müll. Arg.) Vězda

Fellhanera lisowskii (Vězda) Vězda

Fellhanera longispora R. Lücking

Fellhanera cf. misionensis Ferraro \& Lücking ined.

Fellhanera muhlei R. Lücking

Fellhanera paradoxa (Vězda) Vězda

Fellhanera pilomarginata R. Lücking

Fellhanera rhapidophylli (Rehm) Vězda 
Fellhanera rubida (Müll. Arg.) R. Lücking Fellhanera santessonii Barillas \& Lücking Fellhanera stanhopeae (Müll. Arg.) Lück ng et al. Fellhanera subternella (Nyl.) Vězda

Fellhanera tricharioides R. Lücking ined.

Fellhanera verrucifera $\mathrm{R}$. Lück ng

\section{MELANOMMATALES: ASPIDOTHELIACEAE}

Aspidothelium arachnoideum $\mathrm{R}$. Lücking ined. Aspidothelium fugiens (Müll. Arg.) R. Sant.

Aspidothelium geminiparum (Malme) R. Sant. Aspidothelium papillicarpum R. Liicking ined.

\section{MELANOMMATALES: MONOBLASTIACEAE}

Anisomeridium foliicola R. Sant. \& Tibell

Anisomeridium sp. A

Anisomeridium sp. B

Musaespora kalbii R. Lück ng \& Sérus.

\section{MELANOMMATALES: STRIGULACEAE}

Flavobathelium epiphyllum R. Lück ng, Aptroot \& Thor Phyllobathelium anomalum R. Luicking ined. Phyllobathelium epiphyllum (Müll. Arg.) Müll. Arg. Phyllobathelium leguminosae (Cavalc. \& Silva) Lücking \& Sérus.

Strigula antillarum (Fée) Müll. Arg.

Strigula concreta (Fée) R. Sant.

Strigula janeirensis (Müll. Arg.) R. Lück ng

Strigula macrocarpa Vain.

Strigula maculata (Cooke \& Massee) R. Sant.

Strigula microspora R. Lücking

Strigula nemathora Mont.

Strigula nigrocarpa $\mathrm{R}$. Lücking

Strigula nitidula Mont.

Strigula obducta (Müll. Arg.) R. C. Harris

Strigula phyllogena (Müll. Arg.) R. C. Harris

Strigula platypoda (Müll. Arg.) R. C. Harris

Strigula prasina Müll. Arg.

Strigula schizospora R. Sant.

Strigula smaragdula $\mathrm{Fr}$.

Strigula subelegans Vain.

Strigula subtilissima (Fée) Müll. Arg.

Strigula viridis (R. Lücking) R. C. Harris

\section{OSTROPALES: ASTEROTHYRIACEAE}

Asterothyrium cf. anomalum Kalb \& Vězda

Asterothyrium argenteum Müll. Arg.

Asterothyrium aulaxinoides $\mathrm{R}$. Lücking

Asterothyrium chroodisciforme $\mathrm{R}$. Lücking

Asterothyrium hedbergii Kalb \& Vězda

Asterothyrium leucophthalmum (Müll. Arg.) R. Sant.

Asterothyrium microsporum R. Sant.

Asterothyrium monosporum Müll. Arg.

Asterothyrium pittieri Müll. Arg.

Asterothyrium rondoniense Bat. \& Maia ex Henssen \& R.

Lücking

Asterothyrium tetrasporum R. Liicking

Asterothyrium uniseptatum $\mathbf{R}$. Lücking

Gyalidea epiphylla Vězda
Psorotheciopsis philippinensis (Rehm) R. Lücking Psorotheciopsis albomaculans (Rehm) R.. Sant. Psorotheciopsis patellarioides (Rehm) R. Sant. Psorotheciopsis premneella (Müll. Arg.) R. Sant.

\section{OSTROPALES: GOMPHLLACEAE}

Actinoplaca strigulacea Müll. Arg.

Aulaxina dictyospora $\mathrm{R}$. Sant.

Aulaxina intermedia R. Lücking

Aulaxina microphana (Vain.) R. Sant.

Aulaxina minuta R. Sant.

Aulaxina opegraphina Fée

Aulaxina quadrangula (Stirt.) R. Sant.

Aulaxina caleniopsidoides $\mathrm{R}$. Lücking \& Cáceres ined.

Bullatina aspidota (Vain.) Vězda \& Poelt

Calenia chroodisciformis $\mathbf{R}$. Lücking

Calenia conspersa (Stirt.) R. Sant.

Calenia echinoplacoides R. Lücking \& Cáceres ined.

Calenia graphidea Vain.

Calenia lobulata $\mathrm{R}$. Lücking

Calenia monospora Vězda

Calenia phyllogena (Müll. Arg.) R. Sant.

Calenia rolandiana Hartmann

Calenia solorinoides $\mathrm{R}$. Lücking

Calenia thelotremella Vain.

Calenia triseptata Zahlbr.

Caleniopsis laevigata (Müll. Arg.) Vězda

Echinoplaca cf. atrofusca R. Sant.

Echinoplaca bispora Kalb \& Vězda

Echinoplaca campanulata Kalb \& Vězda ined.

Echinoplaca diffluens (Müll. Arg.) R. Sant.

Echinoplaca epiphylla Fée

Echinoplaca furcata Sérus.

Echinoplaca gemmifera R. Liicking

Echinoplaca hymenocarpoides (Vain.) R. Lücking

Echinoplaca cf. intercedens Vězda

Echinoplaca leucotrichoides (Vain.) R. Sant.

Echinoplaca marginata R. Lücking

Echinoplaca melanotrix R. Lücking

Echinoplaca pellicula (Müll. Arg.) R. Sa t.

Echinoplaca tricharioides Kalb \& Vězda

Echinoplaca triseptata R. Lücking

Gyalectid um catenulatum (Cavalc. \& Silva) Ferraro et al.

Gyalectidium eskuchei Sérus.

Gyalectidium filicinum Müll. Arg.

Gyalectidium imperfectum Vězda

Gyalideopsis intermedia $\mathrm{R}$. Lücking

Gyalideopsis rubescens Vězda

Gyalideopsis vulgaris (Müll. Arg.) R. Lücking

Gyalideopsis sp.

Paratricharia paradoxa (R. Lück ng) R. Lücking

Tricharia albostrigosa R. Sant.

Tricharia amazonum Zahlbr.

Tricharia carnea (Müll. Arg.) R. Sant.

Tricharia heterella (Stirt.) R. Lücking

Tricharia hyalina Kalb \& Vězda

Tricharia lancicarpa Kalb \& Vězda

Tricharia longispora Kalb \& Vězda

Tricharia planicarpa R. Lücking

Tricharia helminthospora R. Sant.

Tricharia urceolata (Müll. Arg.) R. Sant.

Tricharia vainioi $\mathrm{R}$. Sant. 


\section{TRICHOTHELIALES: TRICHOTHELIACEAE}

Porina andreana R. Lücking \& Vězda

Porina annulata Schubert, Greber \& R. Lücking ined.

Porina aff. aspera Vězda

Porina atriceps (Vain.) Vain.

Porina atrocoerulea Müll. Arg.

Porina atropunctata R. Lücking \& Vězda

Porina conspersa Malme

Porina curtula Malme

Porina epiphylla (Fée) Fée

Porina fusca R. Lücking

Porina guianensis $\mathrm{R}$. Lücking \& Vězda

Porina imitatrix Müll. Arg.

Porina leptosperma Müll. Arg.

Porina leptospermoides Müll. Arg.

Porina limbulata (Kremp.) Vain.

Porina lucida R. Sant.

Porina mirabilis R. Lücking \& Vězda

Porina nitidula Müll. Arg.

Porina octomera (Müll. Arg.) F. Schill.

Porina papillifera (Stirt.) F. Schill.

Porina pseudoapplanata R. Lücking \& Cáceres

Porina radiata $\mathrm{R}$. Lücking, Kalb \& Vězda

Porina repanda (Stirt.) R. Lücking \& R. Sant ined.

Porina rubentior (Stirt.) Müll. Arg.

Porina rubescens (R. Lücking) Hafellner \& Kalb

Porina rubrosphaera R. Sant

Porina rufula (Kremp.) Vain.

Porina subepiphylla R. Lücking \& Vězda

Porina tetramera (Malme) R. Sant.

Porina triseptata (V̌̌zda) R. Lücking

Porina vezdae R. Lücking

Trichothelium alboatrum Vain.

Trichothelium annulatum (Karst.) R. Sant.

Trichothelium bipindense F. Schill.

Trichothelium epiphyllum Müll. Arg.

Trichothelium juruense (P. Henn.) F. Schill.

Trichothelium longisporum R. Lücking

Trichothelium minus Vain.

Trichothelium minutum (R. Lücking) R. Lücking

Trichothelium mirum $\mathrm{R}$. Lücking

Trichothelium pallescens (Müll. Arg.) F. Schill.

Trichothelium pallidesetum $\mathrm{R}$. Lücking

Trichothelium porinoides Vězda

Trichothelium sipmanii R. Lücking

Trichothelium ulei (P. Henn.) Höhnel

\section{VERRUCARIALES: VERRUCARIACEAE}

Mazentina cererensis R. Lücking ined.

Mazentina perminuta Vězda

primario fueron encontradas 221 especies. Los diez taxones más abundantes presentes en los 100 forófitos del bosque primario son Gyalectidium filicinum, Sporopodium leprieurii, Tricharia vainio, T. urceolata, Dimerella epiphylla, Porina epiphylla, Phyllobathelium epiphyllum, y Anisomeridium foliicola, los
Mazentina sp.

Phylloblastia amazonica Kalb \& Vězda

Pocsia septemseptata Vězda

\section{BASIDIOLICHENES}

Dictyonema phyllogena (Müll. Arg.) Zahlbr.

\section{LICHENES IMPERFECTI}

Phyllophiale alba R. Sant.

Phyllophiale fusca R. Sant.

Phyllophiale viridis.R.Lücking \& Cáceres

Hongos lichenícolas:

\section{ARTHONIALES: ARTHONIACEAE}

Arthonia cinnabarinula Müll. Arg.

Arthonia cryptotheciae R. Sant. ex Matzer \& R. Sant.

\section{ARTHONIALES: OPEGRAPHACEAE}

Opegrapha sipmanii Matzer

\section{DOTHIDEALES: DACAMPIACEAE}

Pyrenidium santessonii R. Lücking Pyrenidium sporopodiorum Matzer Pyrenidium zamiae Matzer

\section{DOTHIDEALES: HYSTERIACEAE}

Hemigrapha tenellula (Müll. Arg.) R. Sant. ex Matzer Hemigrapha strigulae Matzer

\section{DOTHIDEALES: MYCOSPHAERELLACEAE}

Stigmidium porinae Matzer

\section{OSTROPALES: GOMPHILLACEAE}

Gyalideopsis cochlearifer Sérus. \& R. Lücking Gyalideopsis parvula Hafellner \& Vězda

\section{SPHAERIALES: NECTRIACEAE}

Nectria sp.

isidios de tipo Phyllophiale alba, y los campilidios de tipo Calopadia (Cuadro 2A). Si se considera la frecuencia relativa de los diversos tipos de forófitos, así como la proporción de área cubierta por sotobosque, claros y dosel (Cuadro 3), el resultado es diferente (Cuadro 2B). Aunque Gyalectidium filicinum, 
Sporopodium leprieurii y Tricharia vainioi se mantienen como especies más comunes, aparecen con maior frecuencia Porina epiphylla, $P$. rubentior, $P$. mirabilis, $P$. fusca, $P$. radiata, Trichothelium epiphyllum, Mazosia phyllosema, Arthonia leptosperma, y Strigula phyllogena, ya que son las especies más frecuentes en el sotobosque.
Basándose en la frecuencia proyectada a partir de los 100 forófitos investigados, la distribución de frecuencias sigue un patrón exponencial: la mitad de las especies son escasas, creciendo en $3 \%$ o menos de los forófitos (Fig. 2). A esto hay que agregar las 59 especies que no fueron encontradas en los 100 forófitos y que supuestamente son aún más raras.

\section{CUADRO 2}

(A) Frecuenc a relativa de especies de líquenes folícolas sobre los 100 forófitos recolectados, y (B) frecuencia relativa proyectada tomando en cuenta la abundancia relativa de forófitos y la relación de área entre sotobosque, claros y dosel (vea cuadro 2). Se indica las 25 especies más frecuentes en cada caso

(A) Relative frequency of foliicolous lichens species on the 100 selected phorophytes, and (B) projected relative frequency considering the relative abundance of phorophytes and the area relation between understory, light gaps, and canopy (see Table 2). The 25 most frequent species in both cases are indicated

(A) Frecuencia relativa

sobre los 100 forófitos seleccionados
(B) Frecuencia relativa proyectada sobre todos los forófitos del área de estudio
Gyalectidium filicinum

Sporopodium leprieurii

Tricharia vainioi

Phyllophiale alba

Calopadia sp. (campylidia)

Tricharia urceolata

Dimerella epiphylla

Phyllobathelium epiphyllum

Porina epiphylla

Anisomeridium folicola

\section{Aulcxina minuta}

Phylloblastia amazonica

Echinoplaca leucotrichoides

Echinoplaca verrucifera

Aspidothelium fugiens

Porina subepiphylla

Trichothelium epiphyllum

Strigula phyllogena

Actinoplaca strigulacea

Echinoplaca epiphylla

Mazosia phyllosema

Por na mirabilis

Strigula smaragdula

Mazosia rotula

Calopadia puiggarii

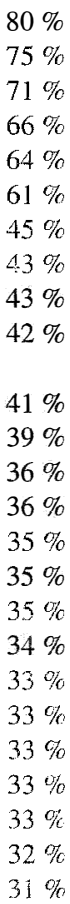

Gyalectiditu filicinum

$29 \%$

Sporopodium leprieurii $\quad 23 \%$

Tricharia vainioi $22 \%$

Calopadia sp. (campylidia) $\quad 21 \%$

Porina epiphylla $\quad 19 \%$

Phyllophiale alba $\quad 18 \%$

Trichotheliun epiphyllum $17 \%$

Mazosia phyllosema $\quad 16 \%$

Tricharia urceolata $\quad 16 \%$

Arthonia leptosperma $\quad 15 \%$

Anisomeridium foliicola $\quad 15 \%$

Phylloblastia amazonica $\quad 14 \%$

Porina rubentior $\quad 14 \%$

Str gula phyllogena $\quad 14 \%$

Porina mirabilis $\quad 13 \%$

Porina fusca $\quad 13 \%$

Porina radiata $13 \%$

Phyllobathelium epiphyllum $\quad 12 \%$

Bapalmuia lineata $\quad 12 \%$

Dimerella siquirrensis $\quad 12 \%$

Phyllophiale fisca $\quad 11 \%$

Por na l mbulata $\quad 11 \%$

Aulaxina minuta $\quad 11 \%$

Dimerella epiphylla $\quad 11 \%$

Calopadia puiggarii $\quad 11 \%$ 


\section{CUADRO 3}

Abundancia relativa de diversos tipos de forófitos en transectos hechos en los senderos CES, CCL, y SHO, y relación de área entre sotobosque, claros y dosel

Relative abundance of different phorophyte types in transects placed along the trails CES, CCL, and SHO, and relative area covered by understory, light gap, and canopy phorophytes

Micrositio:

Relación de área:

Sotobosque

$62 \%$

Tipo de forófito:

Abundancia relativa:

Porcentaje de forófitos con cobertura de líquenes folícolas de más de $20 \%$

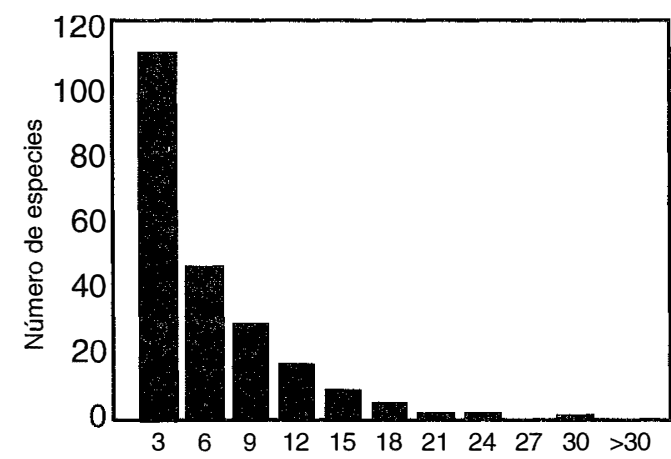

Frecuencia proyectada [\% de forófitos colonizados]

Fig. 2. Distribución de frecuencias de las 221 especies de líquenes folícolas encontradas sobre los 100 forófitos del bosque primario.

Patrones de diversidad $\alpha, \beta, \gamma:$ La diversidad promedia de especies por forófito, o diversidad $\alpha$, varía significativamente entre los tres tipos de micrositios (test de Kruskal \& Wallis: $\mathrm{H}=35.1, \mathrm{p}<0.001)$ : es más alta en el sotobosque (35 especies/forófito) que en los claros (30), y relativamente baja en el dosel (18; Fig. 3). El número total de especies por tipo de micrositio, o diversidad $\gamma$, se comporta parcialmente diferente: es más alta en los claros (169 especies/micrositio) que en el sotobosque (157), pero también baja en el dosel (65). Esto indica una diversidad $\beta$ más alta en los claros en comparación con el sotobosque, lo que significa que los forófitos en los claros son menos homógenos en su composición de

\begin{tabular}{cccccc}
\multicolumn{3}{c}{ Claros } & & \multicolumn{3}{c}{ dosel } \\
& $5 \%$ & & & $33 \%$ & \\
Dicot. & Palmas & Otros & Dicot. & Palmas & Otros \\
$52 \%$ & $25 \%$ & $23 \%$ & $95 \%$ & $4 \%$ & $1 \%$ \\
& & & & & \\
$76 \%$ & $75 \%$ & $80 \%$ & $26 \%$ & - & -
\end{tabular}

líquenes folícolas que los forófitos en el sotobosque.

La diversidad es mayor en las palmas del sotobosque $(37.2 \pm 10.2)$ en comparación con las dicotiledóneas $(33.4 \pm 6.7)$ pero menor en las palmas de los claros $(27.9 \pm 10.7)$ vs. las dicotiledóneas (32.4 \pm 6.8$)$. Sin embargo, estas diferencias no son significativas (test de Mann-Whitney: $U=157, p=0.244$ para el sotobosque; $\mathrm{U}=143.5, \mathrm{p}=0.126$ para los claros). En ambos casos, la variabilidad en la diversidad $\alpha$ (deviación estándar) es más grande en palmas que en dicotiledóneas (10.2-10.7 vs. 6.7-6.8). No hay una diferencia entre las dicotiledóneas del sotobosque y los claros mientras que la diferencia en relación con las palmas es

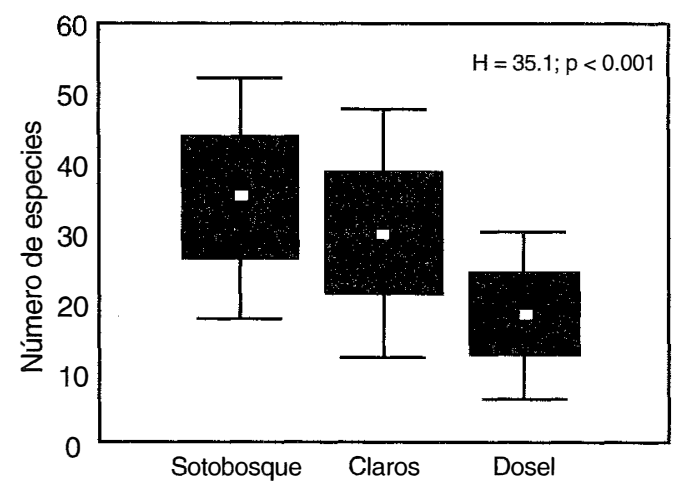

Fig. 3. Comparación del número de especies por forófito (:diversidad $\alpha$ ) en el sotobosque, en los claros y en el dosel, indicando los promedios (puntos) y los intervalos de $65 \%$ (cajas) y $95 \%$ (lineas). 
significativa (test de Mann-Whitney: $\mathrm{U}=103$, $p=0.009$ ), así que la diferencia entre sotobosque y claros en la diversidad se debe principalmente a las palmas.

Agrupación de forófitos: Los dos dendrogramas muestran un patrón muy semejante (Fig. 4A-B). Los forófitos forman 3 grupos separados que corresponden a los tres tipos de micrositios: sotobosque, claros y del dosel, indicando diferencias significativas en la composición de especies de líquenes folícolas en cada tipo de micrositio. En cuanto a los tipos de forófitos (dicotiledoneas vs. palmas), sólo se forman algunos grupos pequeños: se notan seis grupos de dicotiledóneas de 3-5 miembros cada uno, mientras que las palmas forman dos grupos de 4 y 6 miembros. Los dos forófitos del sotobosque que se encuentran en el grupo de los claros (Fig. 4B) son palmas. Aparentemente, entre dicotiledóneas hay más semejanzas en la composición de especies que entre palmas, y en algunas palmas el tipo de forófito es más importante que el microclima.
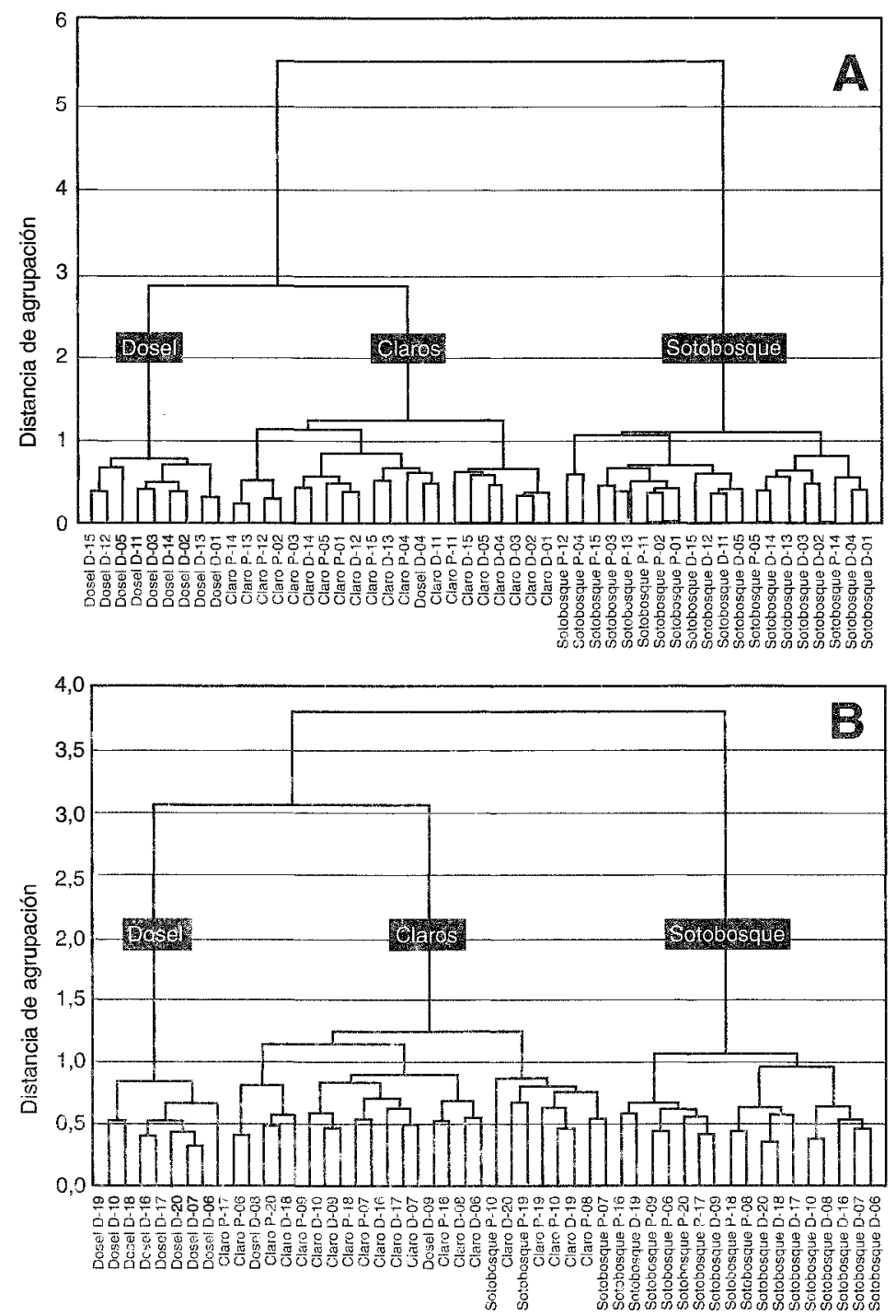

Fig. 4. Dendrogramas del análisis cluster de los 100 forófitos del bosque primario, basados en la composición de especies de líquenes folícolas, separados en dos grupos de 50 forófitos cada uno $(\mathrm{A}, \mathrm{B})$. $\mathrm{D}$ = dicotiledónea; $\mathrm{P}$ = palma (con los números individuales indicados). 
Preferencias de micrositios: En cuanto a sus preferencias por micrositios, las especies pueden ser divididas en seis grupos (Cuadro 4). El grupo 1 consiste en especies que crecen con alta frecuencia sobre forófitos del sotobosque pero son raras en los otros micrositios, mientras que las especies del grupo 2 son intermedias entre sotobosque y claros. Estos dos grupos son dominados por las familias Arthoniaceae, Opegraphaceae, Pilocarpaceae, Strigulaceae (especies supracuticulares), y Trichotheliaceae. El grupo 3 está formado por es- pecies con alta frecuencia en claros, y en el grupo 4 se encuentran especies con transición al dosel. Ambos grupos tienen en común especies de las familias Ectolechiaceae, Gomphillaceae, y Strigulaceae (especies subcuticulares). Finalmente, el grupo 5 está formado por especies con alta frecuencia en o restrictas al dosel. Destácanse en este último grupo las familias Asterothyriaceae y Gomphillaceae.

Las cinco especies del grupo 6 son indiferentes, entre ellas cuatro de la familia Gomphillaceae: Actinoplaca strigulacea, Gyalectidium

\section{CUADRO 4}

Especies de líquenes folícolas con preferencias significativas por diferentes micrositios, comprobados por una prueba de Chi-Cuadrado.

Foliicolous lichen species with significant preferences towards different microsites (Chi-Square-test).

Grupo 1:

Características del sotobosque

Amazonomyces farkasiae

Arthonia accolens

Arthonia leptosperma

Arthonia palmulacea

Aspidothelium papillicar pum

Bapalmuia lineata

Bapalmuia palmularis

Badimia dimidiata

Byssolecania deplanata

Byssolecania fumosonigricans

Byssoloma minutissimum

Cryptothecia candida

Dimerella flavicans

Dimerella siquirrensis

Fellhanera badimioides

Fellhanera rhapidophylli

Fellhanera santessonii

Flavobathelium epiphyllum

Gyalidea epiphylla

Mazosia melanophthalma

Mazosia phyllosema

Mazosia pilosa

Mazosia tenuissima

Microtheliopsis uleana

Paratricharia paradoxa

Porina atropunctata

Phylloblastia amazonica

Phyllophicle fusca

Porina epiphylla

Porina fusca

Porina imitatrix

Porina limbulata

Porina lucida

Porina mirabilis

Porina radiata

Porina rubentior
Porina rufula

Strigula maculata

Strigula phyllogena

Strigula platypoda

Tricharia heterella

Tricharia hyalina

Trichothelium annulatum

Trichothelium epiphyllum

Trichothelium pallescens

Trichothelium ulei

Grupo 2:

Intermedias entre sotobosque y claros

Anisomeridium foliicola

Arthonia aciniformis

Aulaxina minuta

Byssoloma leucoble pharum

Calenia graphidea

Calenia phyllogena

Calopadia phyllogena

Chroodiscus coccineus

Chroodiscus australiensis

Eremothecella calamicola

Fellhanera lisowskii

Gyalideopsis vulgaris

Mazosia rotula

Phyllophiale alba

Phyllobathelium epiphyllum

Porina leptosperma

Porina subepiphylla

Porina tetramera

Sporopodium antonianum

Sporopodium leprieurii

Strigula nemathora

Trichothelium bipindense

Trichothelium minutum
Grupo 3:

Características para claros

Bacidina scutellifera

Byssoloma chlorinum

Calenia depressa

Calopadia foliicola

Calopadia fusca

Echinoplaca epiphylla

Echinoplaca leucotrichoides

Echinoplaca pellicula

Gyalectidium imperfectum

Loflammia flammea

Tapellaria epiphylla

Phyllobathelium leguminosae

Porina atrocoerulea

Porina nitidula

Sporopodium citrinum

Strigula schizospora

Tricharia carnea

Tricharia planicarpa

Trichothelium sipmanii

Grupo 4:

Intermedias entre claros y dosel

Asterothyrium microsporum

Aulaxina opegraphina

Aulaxina quadrangula

Bacidina apiahica

Calopadia puiggarii

Dimerella epiphylla

Echinoplaca hymenocarpoides

Echinoplaca verrucifera

Fellhanera bouteillei

Lasioloma arachnoideum

Strigula antillarum

Strigula nitidula

Strigula smaragdula

Tapellaria nana 
Grupo 5:

Características para dosel

Asterothyrium argenteum Asterothyrium leucopthalmum Asterothyrium monosporum Asterothyrium pittieri Asterothyrium rondoniense Asterothyrium tetrasporum

\author{
Asterothyrium uniseptatum \\ Bullatina aspidota \\ Calenia solorinoides \\ Echinoplaca triseptata \\ Gyalectidium catenulatum \\ Pseudocalopadia mira \\ Psorotheciopsis patellarioides \\ Psorotheciopsis philippinensis \\ Psorotheciopsis premneella
}

Grupo 6:

Especies Indiferentes

Aspidothelium fugiens

Actino placa strigulacea

Gyalectidium filicinum

Tricharia urceolata

Tricharia vainioi filicinum, Tricharia urceolata, y T. vainioi. Estas especies se pueden considerar como generalistas. El resto de las especies, que conforman más de la mitad de la diversidad total, no demuestran preferencias significativas porque son demasiado raras.
Preferencias de forófitos: De los 221 táxones encontrados en los 100 forófitos, solamente 26 muestran diferencias significativas en sus frecuencias sobre dicotiledóneas o palmas (Cuadro 5), así confirmando el bajo grado de preferencias forofíticas obvio en los

\section{CUADRO 5}

Especies de líquenes folícolas con preferencias por dicotiledóneas o palmas, dependiendo de su presencia en el sotobosque y en claros. Especies sin paréntesis tienen preferencias significativas, especies en paréntesis tienen tendencias claras pero no significativas

Foliicolous lichen species with preferences towards palms or dicots, depending on their occurrence in the understory and in light gaps. Species without brackets show significant preferences, species in brackets exhibit clear tendencies which are not significant

Sotobosque

Dicotiledoneas:

[Flavobathelium epiphyllum]

Strigula janeirensis

[Strigula maculata]

Trichothelium annulatum

Trichothelium juruense

Palmas:

Arthonia palmulacea

[Byssolecania hymenocarpa]

[Byssoloma aurantiacum]

[Byssoloma minutissimum]

Byssoloma wettsteinii

Calenia triseptata

Chroodiscus coccineus

Eremothecella calamicola

Fellhanera badimioides

Fellhanera lisowskii

[Fellhanera pilomarginata]

[Fellhanera rhapidophylli]

Fellhanera santessonii

[Mazosia dispersa]

[Paratricharia paradoxa]

[Sporopodium antonianum]

Tricharia heterella

Tricharia hyalina

Tricharia helminthos pora

Tricharia urceolata

[Trichothelium minutum]
Claros

Dicotiledóneas:

Asterothyrilm microsporum

Bacidina scutellifera

Byssoloma leucoblepharum

[Dimerella isidiifera]

Echinoplaca furcata

Loflammia flammea

Mazentina cererensis

[Porina limbulata]

Porina nitidula

[Strigula nemathora]

[Strigula nitidula]

[Strigula phyllogena]

Strigula smaragdula

Palmas:

Actinoplaca strigulacea

Aulaxina microphana

[Byssoloma chlorinum]

[Gyalectidium imperfectum]

Tapellariopsis octomera

[Tricharia planicarpa]

[Trichothelium bipindense] 
dendrogramas. De estas 26 especies, 15 prefieren forófitos del sotobosque y 11 forófitos de claros. Mientras especies que suelen preferir dicotiledóneas en el sotobosque son escasas, 12 especies prefieren palmas en el sotobosque: Arthonia palmulacea, Byssoloma wettsteinii, Calenia triseptata, Chroodiscus coccineus, Eremothecella calamicola,. Fellhanera badimioides, F. lisowskii, F. santessonii, Tricharia helminthospora, T. heterella, T. hyalina, y $T$. urceolata. Nueve especies muestran tendencias no significativas en relación con estos micrositios. Puede ser destacada la frecuencia de especies de Fellhanera, del grupo de Byssoloma minutissimum, y de Tricharia en este grupo.

En los claros, ocho especies muestran preferencias significativas por dicotiledóneas, mientras ocho tienen tendencias no significativas. Aquí se destaca la frecuencia de especies de Bacidina y Strigula. Sólo tres especies suelen preferir palmas, y cinco más tienen tendencias no significativas en los claros. Por lo tanto, se puede concluir que, aparentemente, las preferencias por diferentes forófitos son más pronunciadas en el sotobosque que en los claros, y en el sotobosque hay más preferencias por palmas mientras que en claros hay más preferencias por dicotiledóneas.

Asociaciones de especies y comunidades: El diagrama de asociaciones muestra tres asociaciones principales y dos subasociaciones (Fig. 5). Los tres asociaciones principales corresponden a los grupos de especies $1,3, \mathrm{y}$ 5 , respectivamente (véase Cuadro 4), o sea a los diferentes micrositios: sotobosque, claros, y dosel. Las dos subasociaciones estan formadas por especies que prefieren hojas de palmas o dicotiledóneas en el sotobosque (véase Cuadro 5), con la asociación sobre palmas siendo relativamente grande. El género Porina [números 92-100, 102-104 en Fig. 5] se destaca como grupo característico en el sotobosque, el género Echinoplaca [79-82] en los claros y el género Asterothyrium [62-67] en el dosel. Dentro de un género, las especies con cuerpos fructíferos más oscuros crecen en micrositios más abiertos, como se puede observar comparando Calenia depressa [75; apote- cios oscuros] con $C$. phyllogena [77] y $C$. graphidea [76; apotecios claros], o Calopadia puiggarii [29; apotecios oscuros] con C. fusca [28; apotecios claros]. Sólo dos especies de Porina, $P$. atrocoerulea [91] y P. nitidula [101], crecen en los claros; ambos tienen peritecios negros.

Las tres especies de Gyalectidium demuestran una diferenciación interesante: $G$. filicinum es generalista, pero más común en sotobosque y claros, mientras que G. imperfectum es más abundante en claros y G. catenulatum está restringida al dosel. En el caso de Trichothelium sipmanii, la forma con esporas 7 septadas es más común en los claros, mientras la forma con esporas 8-11-septadas es más común en el sotobosque.

Indices de micrositios: Las variables microclimáticas tienen gran correlación con los índices de micrositios propuestos por Lücking (1997b), con excepción del sotobosque y el grupo intermedio entre sotobosque y claros que no parecen bien separados (Fig. 6). Con base en estos resultados y considerando especies con una frecuencia alta, se propone una modificación del índice de micrositios para 53 de las 270 especies incluidas en la lista original (Lücking 1997b), mientras que para otras 19 especies se propone por primera vez un índice (Cuadro 6).

Comparación florística de cinco diversos tipos de vegetación: Comparando el sotobosque primario, el margen del bosque primario y el sotobosque secundario, no hay diferencias significativas en la diversidad de especies; incluso, la diversidad parece más alta en el bosque secundario (Fig. 7). En la vegetación antropogénica y especialmente en las parcelas de sucesión la diversidad es drasticamente menor (Fig. 7).

La composición por especies de los diversos tipos de vegetación puede ser proyectada en una línea mediante una ordenación por componentes principales, con el sotobosque primario y el dosel del bosque primario como los extremos (Fig. 8). En esta comparación, el margen del bosque se asemeja a los claros dentro del bosque primario, mientras que la vegetación antropogénica y las parcelas de sucessión se encuentran entre los claros y el dosel. 


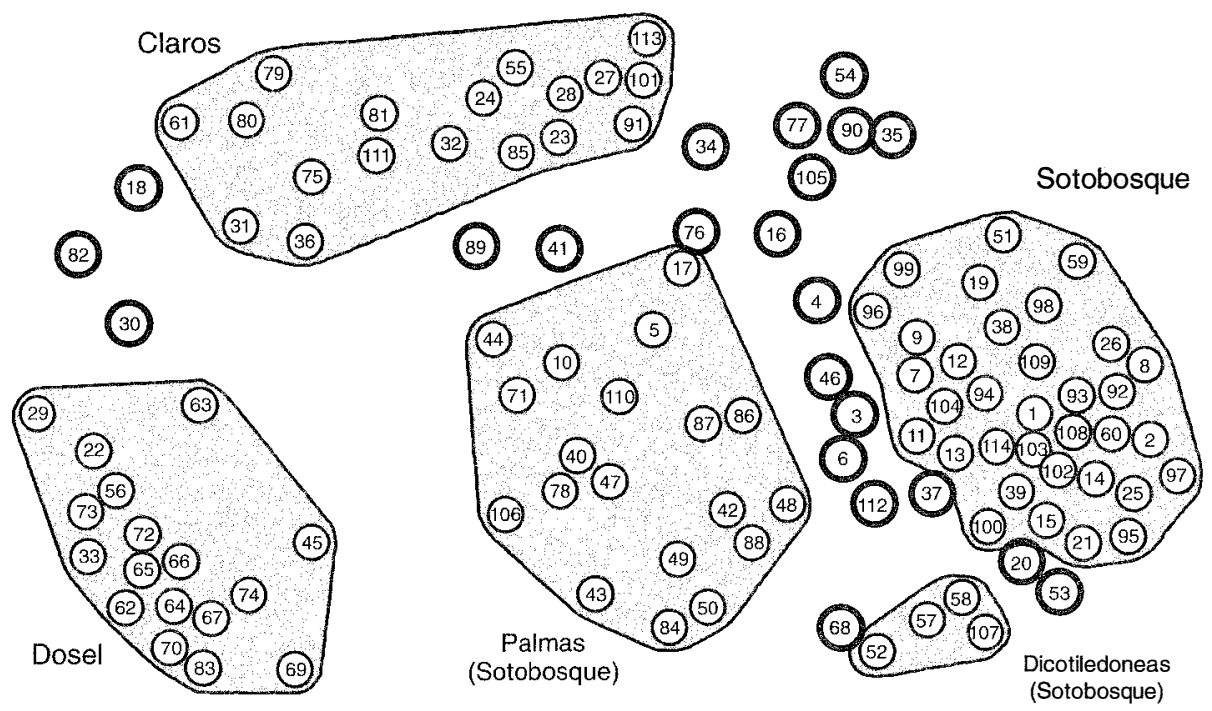

Fig. 5. Diagrama de asociaciones de líquenes folícolas en el bosque primario de La Selva. Las asociaciones y subasociaciones principales que resultan de valores de $X$ stand. > 0.4 (Lücking 1998d) son marcados por áreas rayadas, las especies intermedias por círculos gruesos.

[1] Arthonia accolens

[2] Arthonia leptosperma

[3] Arthonia palmulacea

[4] Cryptothecia candida

[5] Eremothecella calamicola

[6]Mazosia dispersa

[7] Mazosia melanophthalma

[8] Mazosia phyllosema

[9] Mazosia pilosa

[10] Mazosia pseudobambusae

[11] Mazosia rotula

[12] Mazosia rubropunctata

[13] Mazosia tenuissima

[14] Microtheliopsis uleana

[15] Phylloblastia amazonica

[16] Chroodiscus australiensis

[17] Chroodiscus coccineus

[18] Dimerella epiphylla

[19] Dimerella flavicans

[20] Dimerella lisowskii

[21] Dimerella siquirrensis

[22] Bacidina apiahica

[23] Bacidina defecta

[24] Bacidina scutellifera

[25] Bapalmuia lineata

[26] Bapalmuia palmularis

[27] Calopadia foliicola

[28] Calopadia fusca

[29] Calopadia puiggarii

[30] Calopadia sp.

[31] Lasioloma arachnoideum

[32] Loflammia flammea

[33] Pseudocalopadia mira

[34] Sporopodium antonianum

[35] Sporopodium leprieurii

[36] Tapellaria epiphylla

[37] Badimia dimidiata

[38] Byssolecania furnosonigricans
[39] Byssolecania variabilis

[40] Byssoloma aurantiacum

[41] Byssoloma chlorinum

[42] Byssoloma minutissimum

[43] Byssoloma wettsteinii

[44] Fellhanera angustispora

[45] Fellhanera bouteillei

[46] Fellhanera fuscatula

[47] Fellhanera lisowskii

[48] Felhanera pilomarginata

[49] Fellhanera rhapidophylli

[50] Fellhanera santessonii

[51] Anisomeridium foliicola

[52] Flavobathelium epiphyllum

[53] Phyllobathelium anomalum

[54] Phyllobathelium epiphyllum

[55] Phyllobathelium leguminosae

[56] Strigula antillarum

[57] Strigula janeirensis

[58] Strigula maculata

[59] Strigula phyllogena

[60] Strigula platypoda

[61] Strigula schizospora

[62] Asterothyrium argenteum

[63] Asterothyrium microsporum

[64] Asterothyrium monosporum

[65] Asterothyrium pittieri

[66] Asterothyrium tetrasporum

[67] Asterothyrium uniseptatum

[68] Gyalidea epiphylla

[69] Psorotheciopsis patellarioides

[70] Psorotheciopsis premneella

[71] Aulaxina microphana

[72] Aulaxina opegraphina

[73] Aulaxina quadrangula

[74] Bullatina aspidota

[75] Calenia depressa

[76] Calenia graphidea
[77] Calenia phyllogena

[78] Calenia triseptata

[79] Echinoplaca epiphylla

[80] Echinoplaca leucotrichoides

[81] Echinoplaca pellicula

[82] Echinoplaca verrucifera

[83] Gyalectidium laevigatum

[84] Paratricharia paradoxa

[85] Tricharia carnea

[86] Tricharia helminthospora

[87] Tricharia heterella.

[88] Tricharia hyalina

[89] Tricharia planicarpa

[90] Tricharia urceolata

[91] Porina atrocoerulea

[92] Porina atropunctata

[93] Porina epiphylla

[94] Porina guianensis

[95] Porina imitatrix

[96] Porina leptosperma

[97] Porina leptospermoides

[98] Porina limbulata

[99] Porina lucida

[100] Porina mirabilis

[101] Porina nitidula

[102] Porina radiata

[103] Porina rubentior

[104] Porina rufula

[105] Porina subepiphylla

[106] Porina vezdae

[107] Trichothelium annulatum

[108] Trichothelium epiphyllum

[109] Trichothelium minus

[110] Trichothelium minutum

[111] Trichothelium sipmanii

[112] Trichothelium ulei

[113] Phyllophiale alba

[114] Phyllophiale fusca 


\section{CUADRO 6}

(A) Especies de líquenes folícolas con diferencias significativas entre el código de preferencia establecido con base en su distribución en La Selva y el índice de micrositio propuesto por Lücking (1997b), con sugerencias de modificar el índice respectivo. (B) Indices propuestos por primera vez para especies, con base en su distribución en La Selva y observaciones no publicadas. IA = índice propuesto por Lücking (1997b), CP = código de preferencia en La Selva, IM = índice modificado sugerido, $I P=$ indice propuesto por primera vez

(A) Foliicolous lichen species with significant differences between their code of preference, established on base of their distribution at La Selva, and microsite indices as proposed by Lücking (1997b), with suggestions for modifications.

(B) Indices proposed for the first time for species, based on their distribution at La Selva and unpublished observations. $I A=$ index proposed by Lücking (1997b), CP = code of preference at La Selva, IM = suggested modified index,

$I P=$ index proposed for the first time

(A) Indices a modificar

Arthonia palmulacea
Asterothyrium monosporum
Asterothyrium pittieri
Asterothyrium uniseptatum
Bacidina scutellifera
Badimia dimidiata
Bapalmuia palmularis
Byssolecania fumosonigricans
Byssolecania aff. fumosonigricans
Byssoloma chlorinum
Calenia graphidea
Calenia phyllogena
Calopadia phyllogena
Cryptothecia candida
Dimerella dilucida
Dimerella epiphylla
Dimerella siquirrensis
Echinoplaca triseptata
Echinoplaca verrucifera
Fellhanera badimioides
Fellhanera bouteillei
Fellhanera fuscatula
Fellhanera santessonii
Gyalideopsis rubescens
Gyalideopsis vulgaris
Mazosia phyllosema
Mazosia pilosa
Mazosia tenuissima
Phylloblastia amazonica
Phyllophiale fusca
Paratricharia paradoxa
Porina atropunctata

$\begin{array}{lll}\text { IA } & \text { CP } & \text { IM } \\ 2 & 1.0 & 1 \\ 4 & 5.0 & 5 \\ 4 & 4.7 & 5 \\ 4 & 4.8 & 5 \\ 2 & 3.0 & 3 \\ 2 & 1.1 & 1 \\ 2 & 1.3 & 1 \\ 2 & 1.2 & 1 \\ 2 & 1.2 & 1 \\ 4 & 2.6 & 3 \\ 3 & 2.1 & 2 \\ 3 & 2.2 & 2 \\ 3 & 2.1 & 2 \\ 3 & 1.7 & 2 \\ 2 & 1.4 & 1 \\ 2 & 3.3 & 3 \\ 2 & 1.1 & 1 \\ 4 & 5.0 & 5 \\ 3 & 3.8 & 4 \\ 2 & 1.0 & 1 \\ 3 & 4.3 & 4 \\ 1 & 1.8 & 2 \\ 2 & 1.0 & 1 \\ 2 & 3.7 & 3 \\ 3 & 2.2 & 2 \\ 2 & 1.2 & 1 \\ 2 & 1.4 & 1 \\ 2 & 1.3 & 1 \\ 1 & 1.7 & 2 \\ 1 & 1.6 & 2 \\ 2 & 1.0 & 1 \\ 2 & 1.2 & 1\end{array}$

$\begin{array}{llll}\text { Porina nitidula } & 4 & 2.7 & 3 \\ \text { Porina radiata } & 2 & 1.1 & 1 \\ \text { Porina vezdae } & 2 & 2.6 & 3 \\ \text { Sporopodium citrinum } & 4 & 3.0 & 3 \\ \text { Strigula janeirensis } & 2 & 1.3 & 1 \\ \text { Tapellariopsis octomera } & 4 & 3.0 & 3 \\ \text { Tricharia hyalina } & 2 & 1.2 & 1 \\ \text { Trichothelium annulatum } & 2 & 1.0 & 1 \\ \text { Trichothelium bipindense } & 3 & 1.9 & 2\end{array}$

(B) Indices nuevos

IA $\quad$ CP IP

Aspidothelium papillicarpum $\quad-\quad 1.6 \quad 2$ Asterothyrium chroodisciforme $-5.0 \quad 5$

Asterothyrium rondoniense $\quad-\quad 5.0 \quad 5$

Asterothyrium tetrasporum $\quad-\quad 4.7 \quad 5$

Bacidina defecta $\quad-\quad 3.03$

Bapalmuia lineata $\quad-\quad 1.3 \quad 1$

Byssoloma aurantiacum $\quad$ - $\quad \begin{array}{ll}1.8 & 2\end{array}$

Dimerella isidiifera $\quad-\quad 2.4 \quad 2$

Dimerella lisowskii $\quad-\quad \begin{array}{ll}1.2 & 1\end{array}$

Fellhanera rhapidophylli $\quad-\quad \begin{array}{ll}1.6 & 2\end{array}$

Flavobathelium epiphyllum _ $\quad 1.5 \quad 2$

Gyalectidium catenulatum $\quad-\quad 5.0 \quad 5$

Gyalectidium imperfectum _ $3.5 \quad 3$

$\begin{array}{llll}\text { Psorotheciopsis philippinensis } & - & 5.0 & 5\end{array}$

Lyromma palmae $\quad-\quad 1.4 \quad 1$

Mazosia longispora $\quad-\quad \begin{array}{ll}2.3 & 2\end{array}$

Phyllobathelium leguminosae $\quad-\quad 2.7 \quad 3$

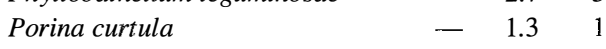

Porina subepiphylla $\quad-\quad 2.12$

Tricharia planicarpa $\quad-\quad 2.5 \quad 2$

Trichothelium juruense $\quad-\quad 1.0 \quad 1$ 


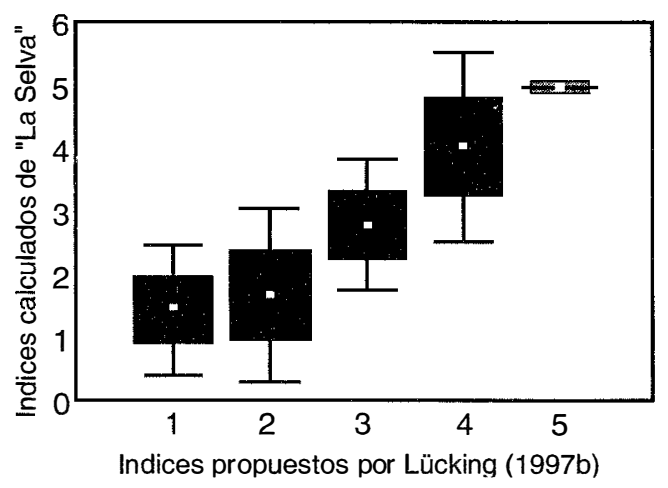

Fig. 6. Comparación de índices de micrositios de especies de líquenes folícolas establecidas en La Selva con los indices propuestos por Lücking (1997b).

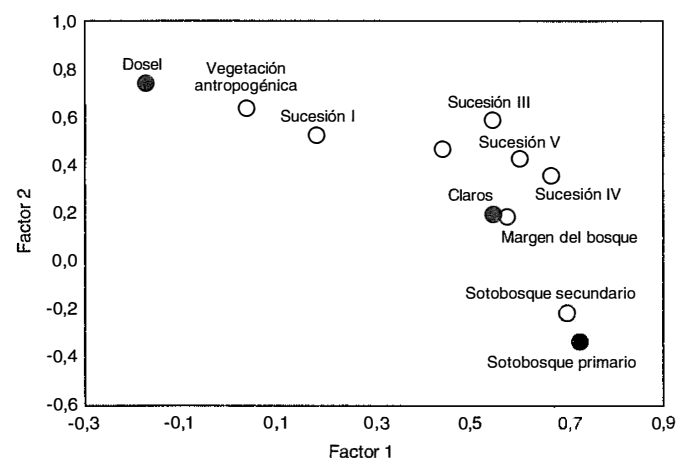

Fig. 8. Ordenación de tipos de vegetación (círculos blancos) y micrositios del bosque primario (círculos negros) basado en un análisis de componentes principales (= factores).

\section{DISCUSIÓN}

El número de 280 especies de líquenes folícolas es el más alto de todos los sitios investigados hasta la fecha, incluso el sendero Botarrama en el parque nacional Braulio Carrillo, donde se encontró 217 especies (Lücking 1999a). Esta diversidad se debe parcialmente a la consideración de especies facultativamente folícolas en los géneros Coccocarpia (2), Coenogonium (3), Cryptothecia (4), Leptogium (1) y Pannaria (1). Sin embargo, aún sin estas especies la diversidad en La Selva sigue siendo excepcionalmente alta, confirmando la hipótesis de que líquenes folícolas son generalmente más diversos en bosques de bajura (Lücking 1995a, 1997b).

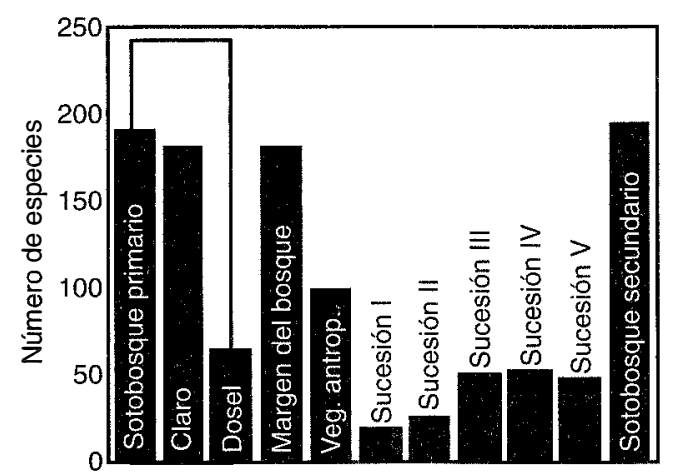

Fig. 7. Diversidad de especies de líquenes folícolas en diversos tipos de vegetación y micrositios en La Selva. La barra blanca sobre los micrositios sotobosque, claros y dosel indica el número total de especies en ellos (= bosque primario).

Las especies encontradas en La Selva significan 80-85\% de todas las especies comunicadas para Costa Rica (Lücking 1995a) y aproximadamente $50 \%$ de las especies conocidas mundialmente (Farkas \& Sipman 1997), así que se destaca como un sitio representativo a nivel mundial para líquenes folícolas. La distribución de frecuencias demuestra la importancia de especies raras para la diversidad, ya que suman más que la mitad del número total.

La Selva probablemente es el sitio mejor estudiado en el Neotrópico en cuanto a la diversidad de especies (McDade et al. 1994). Hasta la fecha se han encontrado alrededor de 1700 especies de plantas vasculares y 705 vertebrados. Para briófitas se puede estimar un número de 200-250 especies, entre ellas 60-80 epífilas (Lücking 1997). Estudios preliminares en líquenes cortícolas en otras regiones (Sipman 1995, Aptroot 1997) sugieren 250-300 especies para un bosque muy húmedo de bajura como La Selva. La presente lista de líquenes folícolas aumenta considerablemente el conocimiento de la diversidad de La Selva. La densidad de especies con $50 \%$ de los taxones conocidos mundialmente es particularmente impresionante cuando se compara con plantas vasculares y vertebrados, donde estos porcentajes alcanzan $0.7 \%$ y $1.6 \%$, respectivamente. Este fenómeno se debe al hecho de que criptógamas no vasculares 
tienen una distribución muy amplia (Grolle 1968, Lücking 1995a).

Una comparación entre La Selva y el sendero Botarrama (Lücking 1999a) muestra un alto grado de similaridad con $80 \%$. La gran mayoría de especies discriminantes entre los dos sitios prefiere zonas bajas o zonas más altas. Especies típicas para el bosque de bajura (Lücking 1997b) y solamente presentes en La Selva incluyen Bacidianigrescens, Bapalmuia spp., Byssolecania spp., Calenia conspersa, Cryptothecia spp., Dimerella hypophylla, Fellhanera badimioides, F. lisowskii, Loflammia gabrielis, Mazosia praemorsa, Porina guianensis, $P$. imitatrix, $P$. papillifera, $P$. radiata, $P$. repanda, Strigula janeirensis, Tricharia amazonum, $T$. carnea, y Trichothelium pallescens. Especies frecuentes en zonas más altas y solamente presentes en el sendero Botarrama son Aspidothelium cinerascens, Byssoloma multipunctata, Calenia subdepressa, Echinoplaca lucernifera, E. serusiauxii, Fellhanera emarginata, Gyalideopsis spp., Tricharia farinosa, T. pseudosantessonii, Trichothelium album, T. echinocarpum, y $T$. poeltii. Esto confirma que el sendero Botarrama en cuanto a su composición de líquenes folícolas es intermedio entre un bosque de bajura y un bosque montano (Lücking 1999a), mientras que la composición de especies en La Selva es típica para un bosque de bajura.

Los patrones de diversidad $(\alpha, \beta, \gamma)$ son comparables con los encontrados en el sendero Botarrama. En particular, la diversidad $\beta$ entre forófitos siempre parece más alta en los claros que en el sotobosque, lo que se explica por el hecho de que la dispersión de líquenes folícolas es más estocástico en los claros y más determinístico en el sotobosque (Lücking 1998a). La principal importancia del microclima para la composición de especies de líquenes folícolas también es consistente con los resultados encontrados en el sendero Botarrama (Lücking 1998b). En general, preferencias por forófitos parecen ser muy sútiles; los patrones más distintos encontrados en La Selva se deben al hecho de que en el presente caso fueron seleccionados solamente dos tipos principales de forófitos, mientras que en el Botarrama se investiga- ron un gran número de forófitos diferentes (Lücking 1998a, 1999a). Obviamente, en el caso de palmas el forófito es más importante que el micrositio, lo que se podría explicar por el hecho de que palmas tienen hojas relativamente grandes que forman un microclima particular menos influenciado por el micrositio. Por eso, en palmas situadas en micrositios más abiertos también pueden establecer líquenes folícolas que normalmente prefieren sitios sombreados. Un fenómeno similar se ha observado en palmas del (sub)dosel, donde la composición de especies es más semejante al sotobosque que a otros forófitos del dosel (Lücking 1995b, Sipman 1997).

Las asociaciones de líquenes folícolas y su composición corresponden en muchos detalles a los encontrados en el sendero Botarrama y en otras investigaciones (Lücking 1995b, 1999b; Lücking et al. 1998b). Generalmente, se puede observar tres asociaciones principales: (1) en el sotobosque, dominadas por Arthoniaceae, Opegraphaceae, Trichotheliaceae y especies supracuticulares del género Strigula; (2) en los claros, dominadas por Gomphillaceae y Ectolechiaceae; (3) en el dosel, dominadas por Asterothyriaceae. Además se forman dos subasociaciones en hojas de palmas del sotobosque (con transición a claros), dominados por Pilocarpaceae y algunas especies de Arthoniaceae, Opegraphaceae y Gomphillaceae, y en hojas dicotyledoneas de claros, dominados por especies subcuticulares del género Strigula. Las especies de las diferentes asociaciones demuestran diferentes características que se puede interpretar como adaptaciones. Por ejemplo, el color de los cuerpos fructíferos es considerado como una adaptación a la intensidad de luz, como protección contra la irradiación, siendo generalmente pálido a transparente en el sotobosque y oscuro en sitios abiertos (Lücking 1999b). La preferencia de especies subcuticulares del género Strigula por hojas dicotiledoneas es un fenómeno conocido (Santesson 1952, Nowak y Winkler 1970, Barillas et al. 1993, Lücking 1998b), y su crecimiento subcuticular también explica su abundancia en claros, por la protección que tienen estas espe- 
cies por su cobertura con la cutícula de la hoja (Lücking 1992a, 1999b).

Las asociaciones del sotobosque y del dosel son más densas que la de los claros. Esto se debe a la distribución de micrositios, que es continuo en el sotobosque y el dosel y necesariamente discontinuo entre los claros. Por eso, la dispersión de especies es más estocástica entre los claros y más determinística dentro del sotobosque y dosel, lo que reduce la probabilidad de encontrar una especie en un claro determinado y así resulta en valores de $\chi_{\text {stand. }}$ más bajas y en asociaciones menos densas (Lücking 1999b).

La observación de que la diversidad de especies parece más alta en el bosque secundario es un fenómeno que se puede observar en muchos organismos y se debe al hecho de que en el bosque secundario la diversidad es concentrada en el tiempo y espacio. Por ser más abierto y más variado en su estructura, en el sotobosque secundario se puede encontrar especies que en el bosque primario sólo crecen en claros o en el dosel. Tomando en consideración todos los micrositios (sotobosque, claros y dosel), la diversidad de especies es significativamente más alta en el bosque primario; sin embargo, esta diversidad es más dificil de reconocer y necesita más esfuerzos en cuanto al tiempo y las técnicas de recolección. La baja diversidad en la vegetación antropogénica y especialmente en las parcelas de sucesión se debe al hecho de que las especies del sotobosque que no soportan una alta intensidad de luz relativa desaparecen en vegetación abierta. $\mathrm{La}$ clasificación de los diversos tipos de vegetación secundaria entre la vegetación primaria demuestra que actualmente no existen especies típicas para vegetación secundaria, muchas veces llamadas especies "ruderales". Aunque en muchos casos son más fáciles de observar en la vegetación secundaria, su hábitat natural suele ser el dosel del bosque primario, donde son difíciles de observar.

La diversidad de líquenes folícolas en un bosque tropical es más alta que lo anteriormente esperado y hasta puede sobrepasar las 300 especies. Junto con briófitas y líquenes cortícolas, el número de especies de criptóga- mas no vasculares en un bosque muy húmedo de bajura como La Selva puede alcanzar 700-800 especies. Además de esta diversidad, destaca la densidad de especies que representa hasta $50 \%$ de la diversidad mundial. Esto significa que sitios como La Selva son representativos para la flora de criptógamas no vasculares tropicales y presentan condiciones ideales para estudiar la ecología de estos organismos.

Las preferencias por micrositios y forófitos de líquenes folícolas son iguales a las encontradas en otros sitios y confirman que estos resultados pueden ser generalizados y representan patrones universales de la ecología de comunidades de líquenes folícolas, particularmente en bosques de ba jura. Entre estos, destacan la formación de tres asociaciones microclimáticas correspondientes al sotobosque, claros y el dosel, y la presencia de una subasociación forofítica en palmas intermedia entre el sotobosque y claros. Para cada asociación se puede identificar géneros característicos: Porina para el sotobosque, Echinoplaca para los claros, Asterothyrium para el dosel, y Fellhanera para las palmas. Estos asociaciones se puede considerar como modelos cuya realización depende de la estructura y dinámica actual de un bosque y de esta manera demuestran ciertas variaciones según el sitio.

El estudio inclusivo de todos los micrositios, junto con el dosel, confirma que el bosque primario soporta la más alta diversidad de especies. Indicaciones de alta diversidad en bosques secundarios que también se conoce de otros organismos se deben al hecho de que en este tipo de vegetación la diversidad se puede observar más facilmente. En el caso de líquenes folícolas, la estructura más abierta de un bosque secundario resulta en la presencia de especies del sotobosque, de claros y del dosel en un área reducida, a veces en una única hoja. Generalmente, la diversidad disminuye en vegetación secundaria y está drasticamente reducida en vegetación abierta. Las especies presentes en este tipo de vegetación no son especies "ruderales" como usualmente se definen, sino que tienen su origen en el dosel del bosque primario. 


\section{AGRADECIMIENTOS}

Agradezco al Servicio de Parques Nacionales y a la Dirección de Vida Silvestre por el permiso para estudiar líquenes folícolas en Costa Rica. Bruce Young amablemente permitió las investigaciones en la Estación Biológica La Selva y se interesó mucho por el presente trabajo. Este estudio fue financiado por la DFG (Deutsche Forschungsgemeinschaft) y el DAAD (Deutscher Akademischer Austauschdienst). También quiero expresar mis gracias a María Isabel Morales, Julieta Carranza y Julián Monge-Nájera y al personal del herbario Nacional, y a Andrea por su apoyo, compañía y paciencia. Dos revisores anónimos hicieron sugerencias valiosas sobre una versión anterior de este trabajo.

\section{REFERENCIAS}

Allan, H. H. 1928. Ecological. In A. Zahlbruckner, K. Keissler \& H. H. Allan. The epiphyllous lichens of Kitchener Park, Feilding, New Zealand. Trans. New Zeal. Inst. 59: 304-314.

Anderson, M. C. 1964. Light relations of terrestrial plant communities. Biol. Rev. 39: 425-486.

Aptroot, A. 1997. Lichen biodiversity in Papua New Guinea, with the report of 173 species on one tree. Bibl. Lichenol. 68: 203-213.

Barillas, R., R. Lücking \& S. Winkler. 1993. Vergesellschaftungen foliikoler Flechten im Biotopo del Quetzal, Guatemala. Cryptogamie Bryol. Lichénol. 14: 49-68.

Busse, W. 1905. Über das Auftreten epiphyllischer Kryptogamen im Regenwaldgebiet von Kamerun. Ber. Deutsch. Bot. Gesellsch. 23: 164-172.

Farkas, E. \& H. J. M. Sipman. 1997. Checklist of foliicolous lichenized fungi. In E. Farkas \& T. Pócs (eds). Cryptogams in the phyllosphere: Systematics, distribution, ecology, and use. Abstr. Bot. 21: 173-206.

Fitting, H. 1910. Über die Beziehungen zwischen den epiphyllen Flechten und den von ihnen bewohnten Blättern. Ann. Jard. Bot. Buitenzorg, ser. 2, suppl. 3: 505-518.
Gauch, H. G. jr. 1982. Multivariate analysis in community ecology. Cambridge University, Cambridge.

Goodall, D. W. 1978. Sample similarity and species correlation. Pp. 99-149, In R. H. Whittaker (ed.). Ordination of plant communities. Junk, The Hague.

Gradstein, S. R. \& T. Pócs. 1989. Bryophytes. Pp. 311325, In H. Lieth \& M. J. A. Werger (eds). Tropical rain forest ecosystems - Biogeographical and ecological studies (Ecosystems of the world 14B). Elsevier, Amsterdam.

Greig-Smith, P. 1983. Quantitative Plant Ecology (Studies in ecology vol. 9). University of California, California.

Grolle, R. 1968. Grossdisjunktionen in Artarealen lateinamerikanischer Lebermoose. Pp. 562-582 In E. J. Fittkau, J. Illies, H. Klinge, G. H. Schwabe \& H. Sioli (eds). Biogeography and ecology in South America. Junk, The Hague.

Hartshorn, G. S. \& B. E. Hammel. 1994. Vegetation types and floristic patterns. Pp. 73-89, In L. A. McDade, K. S. Bawa, H. A. Hespenheide \& G. S. Hartshorn (eds). La Selva. University of Chicago, Chicago.

Jongman, R. H. G., C. J. F. ter Braak \& O. F. R. van Tongeren. 1995. Data analysis in community and landscape ecology. Cambridge University, Cambridge.

Kalb, K. \& A. Vězda. 1988. Neue oder bemerkenswerte Arten der Flechtenfamilie Gomphillaceae in der Neotropis. Bibl. Lichenol. 29: 1-80, 39 pl.

Lücking, R. 1992a. Zur Verbreitungsökologie foliikoler Flechten in Costa Rica, Zentralamerika. Teil I. Nova Hedwigia 54: 309-353.

Lücking, R. 1992b. Zur Verbreitungsökologie foliikoler Flechten in Costa Rica, Zentralamerika. Teil II. Herzogia 9: 81-112.

Lücking, R. 1992c. Foliicolous lichens - A contribution to the knowledge of the lichen flora of Costa Rica, Central America. Beih. Nova Hedwigia 104: 1-179.

Lücking, R. 1995a. Biodiversity and conservation of foliicolous lichens in Costa Rica. In C. Scheidegger, P. A. Wolseley \& G. Thor (eds). Conservation biology of lichenised fungi. Mitteil. Eidgenöss. Forsch.anstalt Wald, Schnee Landsch. 70: 63-92.

Lücking, R. 1995b. Foliikole Flechten auf Cecropiaceen im Kronendach eines tropischen Regenwaldes. Bibl. Lichenol. 58: 261-274. 
Luicking, R. 1995c. Additions and corrections to the foliicolous lichen flora of Costa Rica. The family Arthoniaceae, with notes on the genus Stirtonia. Lichenologist 27: 127-153.

Lücking, R. 1995d. Lista preliminar de líquenes folícolas de las principales areas protegidas de Costa Rica. Brenesia 43-44: 39-46.

Lücking, R. 1997a. Estado actual de investigaciones sobre líquenes folícolas en la región neotrópica, con un análisis biogeográfico preliminar. Trop. Bryol. 13: 87-114.

Lücking, R. 1997b. The use of foliicolous lichens as bioindicators in the tropics, with special reference to the microclimate. In E. Farkas \& T. Pócs (eds). Cryptogams in the phyllosphere: Systematics, distribution, ecology, and use. Abstr. Bot. 21: 99-116.

Lücking, R. 1997c. Additions and corrections to the knowledge of the foliicolous lichen flora of Costa Rica. The family Gomphillaceae. Bibl. Lichenol. 65: 1-109.

Lücking, R. 1997d. Notes on "Lichenes Foliicoli Exsiccati", Fase. I-VI. In E. Farkas \& T. Pócs (eds). Cryptogams in the phyllosphere: Systematics, distribution, ecology, and use. Abstr. Bot. 21: 89-98.

Lücking, R. 1997e. Additions and corrections to the knowledge of the foliicolous lichen flora of Costa Rica. The genus Fellhanera, with notes on Bacidia pauciseptata. Trop. Bryol. 13: 141-173.

Lücking, R. 1998a. Ecology of foliicolous lichens at the "Botarrama" trail (Costa Rica), a neotropical rain forest. II. Patterns of diversity and area cover and their dependence on microclimate and phorophyte species. Ecotropica $4: 1-24$.

Lücking, R. 1998b. Ecology of foliicolous lichens at the "Botarrama" trail (Costa Rica), a neotropical rain forest. III. Phorophyte range and patterns of phorophyte specifity. Phyton (Horn) 38: 195-219.

Lücking, R. 1998c. Additions and corrections to the knowledge of the foliicolous lichen flora of CostaRica. The genus Trichothelium. Nova Hedwigia 66: 375-417.

Lücking, R. 1999a. Ecology of foliicolous lichens at the "Botarrama" trail (Costa Rica), a neotropical rain forest. I. Species composition and its ecogeographical implications. Biotropica (en prensa).

Lücking, R. 1999b. Ecology of foliicolous lichens at the "Botarrama" trail (Costa Rica), a neotropical rain forest. IV. Species associations, their salient features, and their dependence on environmental parameters. Lichenologist 31: 269-289.
Lücking, R., A. Aptroot \& G. Thor. 1997. New species or interesting registros of foliicolous lichens. II. Flavobathelium epiphyllum (Lichenized Ascomycetes: Melanommatales). Lichenologist 29: 221-228.

Lücking, R., U. Becker \& G. Follmann. 1998b. Foliikole Flechten aus dem Taï-Nationalpark, Elfenbeinküste (Tropisches Afrika). II. Ökologie und Biogeografie. Herzogia 13: 207-228.

Lücking, R. \& A. Lücking. 1996. Foliicolous lichens and bryophytes. In P. Hietz, R. Lücking, A. Lücking, H. J. M. Sipman, H. F. M. Vester, H. F. M., J. H. D. Wolf \& E. Gardette. How to sample the epiphytic diversity of tropical rain forests. Ecotropica 2: 59-72.

Lücking, R., T. H. Lumbsch \& J. A. Elix. 1994. Chemistry, anatomy and morphology of foliicolo $\mathrm{s}$ species of the genera Fellhanera and Badimia (Lichenized Ascomycotina: Lecanorales). Bot. Acta 107: 393-401.

Lücking, R. \& M. Matzer. 1996. Ergänzungen und Verbesserungen zur Kenntnis der foliikolen Flechtenflora Costa Ricas. Die Familie Opegraphaceae (einschließlich der Gattung Mazosia). Nova Hedwigia 63: 109-144.

Lücking, R. \& E Sérusiaux. 1997. Musaespora kalbii sp. nov. (Lichenized Ascomycetes: Melanommatales), a new foliicolous lichen with pantropical distribution. Nord. J. Bot. 16: 661-668.

Lücking, R., E. Sérusiaux, L. C. Maia \& E. C. G. Pereira. 1998a. A revision of names of foliicolous, lichenized fungi published by Batista and co-workers between 1960 and 1975. Lichenologist 30: 121-191.

Lücking, R. \& A. Vězda. 1998. Taxonomic studies in foliicolous species of the genus Porina. II. The Porina epiphylla aggregate. Willdenowia 28: 181-225.

Matzer, M. 1996. Lichenicolous Ascomycetes with fissitunicate asci on foliicolous lichens. Myc. Pap. 171: i-x, 1-202.

McDade, L. A., K. S. Bawa, H. A. Hespenheide \& G. S Hartshorn (eds). 1994. La Selva. University of Chicago Press, Chicago.

Nowak, R. \& Winkler, S. 1970. Foliicole Flechten der Sierra Nevada de Santa Marta (Kolumbien) und ihre gegenseitigen Beziehungen. Österr. Bot. Zeitsch. 118: 456-485.

Perry, D.R. \& J. Williams. 1981. The tropical rain forest canopy: a method providing total access. Biotropica 13: $283-285$

Pócs, T. 1978. Epiphyllous communities and their distribution in East Africa. Bryophyt. Bibl. 13: 681-713. 
Richards, P. W. 1984. The ecology of tropical forest bryophytes. Pp. 1233-1270 In R. M. Schuster (ed.). New manual of bryology, vol. 2. Hattori Botanical Laboratory, Nichinan.

Santesson, R. 1952. Foliicolous lichens. I. A revision of the taxonomy of the obligately foliicolous lichenized fungi. Symb. Bot. Ups. 12(1): 1-590.

Sérusiaux, E. 1989. Foliicolous lichens: ecological and chorological data. Bot. J. Linn. Soc. 100: 87-96.

Sérusiaux, E. \& J. R. De Sloover 1986. Taxonomical and ecological observations on foliicolous lichens in northern Argentina, with notes on the hyphophores of Asterothyriaceae. Veröff. Geobot. Inst., Zürich 91: 260-292.

Sipman, H. J. M., 1995. Preliminary review of the lichen biodiversity of the Colombian montane forests. Pp. 313-320 In S. P. Churchill et al. (eds). Biodiversity and conservation of Neotropical montane forests. New York Botanical Garden, New York.
Sipman, H. J. M. 1997. Observations on the foliicolous lichen and bryophyte flora in the canopy of a semideciduous tropical forest. In E. Farkas \& T. Pócs (eds). Cryptogams in the phyllosphere: Systematics, distribution, ecology, and use. Abstr. Bot. 21: 153-161.

Sipman, H. J. M. \& R. C. Harris. 1989. Lichens. Pp. 303309 In H. Lieth \& M. J. A. Werger (eds). Tropical rain forest ecosystems - Biogeographical and ecological studies (Ecosystems of the world 14B). Elsevier, Amsterdam.

Sørensen, T. 1948. A method of establishing groups of equal amplitude in plant sociology based on similarity of species content. Biol. Skrift. K. Danske Vidensk. Selsk. 5: 1-34.

Vareschi, V. 1980. Vegetationsökologie der Tropen. Ulmer, Stuttgart.

Wilbur, R. L. et al. 1994. Vascular plants: An interim checklist. Pp. 350-378, In L. A. McDade, K. S. Bawa, H. A. Hespenheide \& G. S. Hartshorn (eds). La Selva. University of Chicago, Chicago. 